\title{
Bernard Heyberger, Rémy Madinier (éd.), L'Islam des marges. Mission chrétienne et espaces périphériques du monde musulman $\mathrm{XVI}^{\mathrm{e}}-\mathrm{XX}^{\mathrm{e}}$ siècles
}

Paris, IISMM-Karthala, coll. « Terres et gens d'islam », 2011, 285 p.

Catherine Mayeur-Jaouen

\section{(2) OpenEdition}

Édition électronique

URL : http://journals.openedition.org/assr/23503

DOI : $10.4000 /$ assr.23503

ISSN : $1777-5825$

Éditeur

Éditions de l'EHESS

Édition imprimée

Date de publication : 31 décembre 2011

Pagination : 176

ISBN : 9782713223273

ISSN : 0335-5985

\section{Référence électronique}

Catherine Mayeur-Jaouen, «Bernard Heyberger, Rémy Madinier (éd.), L'Islam des marges. Mission chrétienne et espaces périphériques du monde musulman $x \mathrm{vl}^{\mathrm{e}-\mathrm{xx}} \mathrm{x}^{\mathrm{e}}$ siècles », Archives de sciences sociales des religions [En ligne], 156 | octobre-décembre 2011, document 156-53, mis en ligne le 15 février 2012, consulté le 21 septembre 2020. URL : http://journals.openedition.org/assr/23503 ; DOI : https://doi.org/10.4000/assr.23503

Ce document a été généré automatiquement le 21 septembre 2020.

(c) Archives de sciences sociales des religions 


\section{Bernard Heyberger, Rémy Madinier (éd.), L'Islam des marges. Mission chrétienne et espaces périphériques du monde musulman $\mathrm{XVI}^{\mathrm{e}}-\mathrm{XX}^{\mathrm{e}}$ siècles}

Paris, IISMM-Karthala, coll. « Terres et gens d'islam », 2011, 285 p.

Catherine Mayeur-Jaouen

\section{RÉFÉRENCE}

Bernard Heyberger, Rémy Madinier (éd.), L'Islam des marges. Mission chrétienne et espaces périphériques du monde musulman $\mathrm{XVI}^{\mathrm{e}}-\mathrm{XX}^{\mathrm{e}}$ siècles, Paris, IISMM-Karthala, coll. « Terres et gens d'islam », 2011, 285 p.

1 Comme le rappelle l'introduction de cet ouvrage collectif, "l'histoire de la mission chrétienne en islam est d'abord celle d'un échec retentissant » (p. 7), l'islam n'ayant guère été ébranlé par les efforts des missions catholiques, protestantes ou orthodoxes qui se tournèrent alors souvent vers les chrétiens orientaux, à moins qu'elles ne se soient intéressées à eux dès le début. L'existence de communautés musulmanes " hétérodoxes", plus accueillantes ou réputées telles, donna toutefois quelque espoir aux missionnaires qui espérèrent mieux réussir auprès des druzes du Mont-Liban, des alaouites de Syrie, des Ahl-e Haqq du Kurdistan iranien, des musulmans abangan de Java, des Berbères de la Grande Kabylie. C'est l'histoire de ces missions auprès de musulmans jugés à tort ou raison marginaux (géographiquement ou religieusement) qu'aborde ce volume original, clair et d'une lecture agréable.

2 Fruit d'un colloque tenu à Paris en 2007, il réunit neuf contributions couvrant en gros l'époque moderne et contemporaine. La majorité ont pour cadre le $\mathrm{xIx}^{\mathrm{e}}$ siècle et le début $\mathrm{du} \mathrm{Xx}^{\mathrm{e}}$ siècle, époque du renouveau des missions à l'heure coloniale, même si le pouvoir colonial eut plutôt tendance à réfréner les ardeurs missionnaires qu'à les 
favoriser, et contribua souvent à l'islamisation de régions entières plutôt qu'à leur christianisation. Les premières décennies $\mathrm{du} \mathrm{xx}^{\mathrm{e}}$ siècle voient un mouvement d'acculturation et d'indigénisation des missionnaires - ou d'une partie des missionnaires, avec de vives tensions parfois au sein d'une même congrégation - au moment même où le monde musulman, qui se perçoit de plus en plus comme une forteresse assiégée, menacée par la conversion au christianisme, s'applique à redéfinir des normes nouvelles pour permettre la régénération d'un islam jugé abâtardi. Les missionnaires, leurs écrits et leurs écoles n'auront pas peu contribué à diffuser cette condamnation de l'islam populaire, notamment rural, par le réformisme musulman urbain. A fortiori auront-ils contribué à rendre suspects des groupes musulmans marginaux, ou jugés tels, en leur supposant une plus forte propension à se convertir au christianisme, ou en décelant chez eux un hypothétique substrat soit chrétien (les Kabyles, les Alévis, les Ahl-e Haqq), soit païen (les Bamoun du Cameroun, en s'efforçant d'y dégager une "coutume" acceptable par le christianisme), soit hindou (l'Inde moghole, selon un cliché orientaliste très répandu qui voit l'Inde comme un grand socle hindou qu'auraient mal entamé christianisation et islamisation). Le livre montre très bien que les modes de vie montagnards, ruraux, généralement misérables de ces communautés supposées isolées ont joué, tout autant que leur hétérodoxie présumée, dans la construction d'images stéréotypées, souvent élaborées au moment même où ces groupes, précisément, étaient en voie d'intégration: ainsi les Kabyles qui commencèrent à miser sur l'école et l'émigration, grâce aux Pères blancs qui justement espéraient convertir ces communautés jugées immobiles; ainsi les alaouites de Syrie dont certains se convertirent (pas en masse) dans les années 1930 justement au moment où ils accédaient à un meilleur statut social. Le thème prégnant de l'îlot montagnard ou rural conservatoire de coutumes et de pratiques ancestrales, de superstitions à éliminer ou de «coutumes" à préserver éventuellement en les christianisant pose finalement la question plus générale de la misère rurale : a-t-elle des conséquences religieuses? Et quelles sont les relations entre les élites urbaines et la masse paysanne? Rien de particulier aux musulmans, comme le souligne - à peu près seul dans le recueil - Bernard Heyberger qui évoque les "finistères de la catholicité " (Alain Cabantous) auxquels s'attaquent les prêtres catholiques en Europe à l'époque moderne. En somme, l'idée que «le Christ s'est arrêté à Eboli» ou l'immobilisme supposé des campagnes de Donnafugata dans Le Guépard trouvent ici un écho.

3 La marginalité géographique, sociale ou économique de certaines communautés abordées dans le volume ne vaut pas pour les actifs Bamoun, situés sur un carrefour africain ; ou a fortiori pour le petit milieu de lettrés et de poètes qui entoure la Cour de l'Empereur moghol Akbar. À la vérité, l'article de Hugues Didier qui traite des missions jésuites portugaises auprès d'Akbar qui inventa peut-être (et peut-être pas) une nouvelle religion syncrétiste traite d'un tout autre sujet que les autres articles du recueil : il eût fallu pour aborder ce sujet difficile une bibliographie plus solide, plus variée et plus récente que le recours trop systématique à S. A. Rizvi (1975) et K. A. Nizami (1989) - une bibliographie restreinte et nécessairement piégée, en contexte indo-pakistanais, par des enjeux contemporains : H. Didier s'en montre conscient, sans parvenir à s'en libérer par le recours direct aux sources persanes et en se contentant des sources jésuites portugaises. «Les jésuites avaient diagnostiqué la non-conformité d'Akbar avec l'orthodoxie sunnite (...)» (p. 26) : sans doute, mais il faut seulement en conclure que les jésuites avaient une idée dogmatique de ce que devait être l'orthodoxie sunnite. Quant aux rapports entre la supposée hétérodoxie d'Akbar, les 
courants chiites et sunnites alors venus d'Iran, le legs des philosophes musulmans et le monisme d'Ibn 'Arabî, il faudrait pour en traiter avoir lu au moins quelques pans de littérature sur la prophétologie musulmane et la façon dont Jésus ('Isâ) s'y inscrit, ou sur l'antinomisme musulman (se couper les moustaches a une fonction rituelle dans des groupes particuliers, dans la ligne des qalandars) avant de parler de "postchiites » ou de "postmusulmans» (sic), tout en transcrivant muslîm par muslim et mu'min par mu'mîn (resic).

4 L'article de Hugues Didier a au moins le mérite de pointer cette pluralité extrême qui est au fond, et pas seulement en Inde, la réalité massive de l'islam historique, et pas seulement sur ses marges. Florence Hellot conclut à ce même bouillonnement d'idées et de courants religieux dans la Perse du XIX siècle: les périodes de réformes, qui sont aussi des temps de crises, ont sans doute ouvert le champ aux missions plus largement que par le passé, en donnant aussi davantage de curiosité à certains musulmans à l'égard du christianisme et de ce que pouvaient proposer les chrétiens, sans aller en général jusqu'à se convertir. Dans le cas particulier de Java, le christianisme est parvenu à s'implanter directement, peut-être grâce à l'affirmation de la diversité religieuse dans la religion javanaise traditionnelle (hindo-bouddhique, elle-même fruit de métissages complexes) - qu'elle soit islamisée ou non - et dans le Pancasila adopté à l'indépendance et qui reconnaît cinq religions (islam, protestantisme, catholicisme, hindouisme et bouddhisme). Rémy Madinier présente avec vivacité l'islam abangan, version musulmane de la «synthèse mystique » javanaise, les missions catholiques et protestantes, et les oppositions entre cet islam javanais fort accommodant - comme tout l'islam moderne, en fait - et les tenants d'un islam réformiste, celui des santri et des pesantren - soucieux d'épuration de la religion "traditionnelle ", dans le droit fil des réformistes de tout le monde musulman à la fin du XIX ${ }^{e}$ siècle. Au XIX siècle, des conversions de masse au protestantisme sont incontestablement liées à un désir de promotion sociale et politique. Des jésuites à la fin du xix siècle proposaient, au sein d'une même congrégation, deux modèles missionnaires différents, l'un prônant une acculturation en douceur (maintien de la circoncision, acceptation de mariages mixtes), l'autre imposant davantage un modèle de christianisme européen, qui pouvait séduire ceux qui voulaient entrer dans la société européenne par conversion. Alexandra Loumpet-Galitzine consacre un excellent article à la mission protestante face à l'islam bamoun à l'ouest du Cameroun. L'extraordinaire roi bamoun N'joya (vers 1865-1933) dont le musée d'ethnographie de Berlin expose les collections et les photographies non moins extraordinaires, fut l'inventeur d'une religion syncrétique et même d'une écriture, faisant son miel de ses contacts aussi bien avec l'islam peul ou haoussa qu'avec les missions protestantes allemandes (à partir de 1902-1906), puis françaises - sans se convertir pour autant à l'islam ni au christianisme : le patrimoine bamoun incorpora bien des éléments appelés "coutumes" par les missionnaires et l'administration coloniale pour désigner «la transformation d'éléments culturels filtrés en normes acceptables» (p.268), en opérant ainsi une distinction entre sacré et séculier, et en imposant ainsi une norme - d'ailleurs modifiable - à la culture indigène qui veilla à se les réapproprier. Islam et christianisme cohabitaient ainsi, souvent au sein de la même famille, grâce à une métaculture religieuse bamoun supposée supraconfessionnelle. Cette "coutume" avait été créée par les protestants, en principe à partir du paganisme, en réalité souvent à partir de l'islam local, et en général par un procédé d'autoexotisation auquel participèrent pleinement les Bamoun. 
Dans son ensemble, le livre touche à plusieurs sujets fondamentaux : le premier, et non le moindre, qui traverse la plupart des articles est l'apport essentiel des missionnaires à l'orientalisme érudit, particulièrement souligné dans l'article de Bernard Heyberger sur les missions catholiques auprès des druzes et nusayrîs de Syrie aux XVII ${ }^{\mathrm{e}}$ et XVIII ${ }^{\mathrm{e}}$ siècles - qui montre l'apport réel d'informations justes comme celles de Michel Nau sur les nusayris, mais aussi l'influence durable de clichés répétés partout, comme celui des origines franques et croisées des druzes. Les missionnaires comme les intéressés adhéraient-ils à de telles fictions? Il semble, conclut Bernard Heyberger, que «la recherche d'une alliance extérieure contre le pouvoir en place a été un des mobiles principaux du rapprochement entre druzes et missionnaires» (p. 75), ainsi que l'importance de l'alliance des druzes avec les clans maronites. D'autres articles campent davantage les missionnaires en ethnographes de terrain, comme les jésuites dans la montagne alaouite des années 1930: Chantal Verdeil compare la vision des alaouites syriens par les jésuites et les avancées actuelles de la recherche sur ces communautés, sans toutefois établir, et c'est dommage, le rapport avec les Alévis turcs ou iraniens qu'évoquent par ailleurs Kieser ou Membrado. De façon très intéressante, elle pointe également la divergence entre le diagnostic des érudits de la Compagnie (qui continuent à parler d'origines croisées ou de syncrétisme dans des textes publiés) et les lettres non publiées des missionnaires de terrain qui diagnostiquent pour leur part le désir d'émancipation sociale à la base des quelques conversions au christianisme. La différence entre les préjugés despublicistes américains et les critiques antiimpérialistes des missionnaires présents sur place est également soulignée par HansLukas Kieser dans un autre article. Karima Dirèche montre la construction par les orientalistes de l'identité berbère, ainsi que la kabylophilie coloniale, et finalement la façon dont les Pères blancs ont ignoré la vie religieuse des Kabyles (« un islam que l'on ne voit pas », p. 123) en les réduisant à leur misère et à de supposées superstitions. Mojan Membrado est sans doute l'auteur du volume qui évoque le plus directement cet «islam que l'on ne voit pas", pour le comparer aux visions présentées par les missionnaires - des «assemblages hasardeux d'éléments provenant d'une (ou plusieurs) sous-branches pour donner une combinaison de doctrines et pratiques qui ne correspond aux croyances d'aucune d'entre elles » (p. 224), ce qui permet donc aux missionnaires de forger la thèse du cryptochristianisme des Ahl-e Haqq, hélas reprise par de nombreux orientalistes.

6 L'histoire des missions est évidemment, et par définition, un autre aspect important du livre, singulièrement dans l'article synthétique de Hans-Lukas Kieser sur les stratégies des missionnaires américains dans le monde ottoman : après avoir rêvé d'évangéliser les juifs et musulmans du Levant dans les années 1820, l'American Board se tourne dans les années 1830 vers l'idée d'un christianisme régénéré (revival), misant à la fois sur les réformes (Tanzimat) et sur les Arméniens d'Anatolie, toujours dans l'espoir du Millenium imminent. La création d'un millet protestant, puis d'une Constitution des protestants, mise en vigueur en 1856, servit peut-être de référence au mouvement constitutionnel ottoman, tout en attirant l'attention des Alévis kurdes. Mais la stratégie du revival renforçait la jalousie entre les différents groupes confessionnels ou ethniques d'une même région, mettait à distance l'islam sunnite ottoman, se trompait en croyant que l'élite sécularisée sortie des écoles américaines permettrait une sorte de conversion séculière des musulmans, enfin connut un effondrement final face à la destruction des chrétiens d'Asie Mineure lors de la Première Guerre. Florence Hellot-Bellier propose un récit plus narratif des différents groupes missionnaires à l'œuvre en Perse, 
principalement au Kurdistan d'Iran, comme les missionnaires à la rencontre des mystérieux Ahl-e Haqq que présente Mojan Membrado.

7 Un troisième sujet qui traverse tout le livre, associé de près à la question de l'orientalisme, est la circulation de livres ou d'écrits au sein du monde musulman : de nombreux articles évoquent la publication et la diffusion de Bibles ou de Corans, les traductions - notamment celles de la Bible - dans lesquelles les missionnaires jouent un rôle déterminant, enfin l'enjeu fondamental de l'apprentissage des langues pour l'acculturation des uns et des autres. On mesure l'importance des presses missionnaires (en persan, en turc, en syriaque, en arabe...), la manipulation d'imprimés par les missionnaires (contribuant parfois à forger des sources de référence pour les renouveaux culturels locaux) et enfin la production d'écrits spécifiques pour entamer le dialogue avec les musulmans et leur faire connaître le christianisme, avec des débats intéressants qui montrent après tout de vraies rencontres dont le livre donne plusieurs exemples : entre les jésuites portugais et les lettrés de la Cour d'Akbar, entre un carme et le cheikh nusayrî Soliman en 1709, entre missionnaires et mojtaheds iraniens de la fin du XIXe siècle, entre le père jésuite Van Lith et les Javanais qui voyaient en lui un " maître de sagesse »... Le cheikh Ahl-e haqq Jeynûnabâdî (m. 1920), fondateur d'une importante branche réformée à tonalité soufie, possédait sans doute une Bible, ce qui n'en fait pas un chrétien, mais montre une curiosité sans doute assez courante.

Le livre s'inscrit pleinement dans le renouveau de l'historiographie missionnaire, affranchie des frontières nationales ou confessionnelles. Des élargissements sont encore possibles et souhaitables, en particulier vers l'Asie centrale où les missions russes orthodoxes, en lien avec l'orientalisme russe, ont abouti à la création d'un islam des marges (l'islam des steppes souffrant de toutes les tares, à la fois rural, nomade, soufi, rétrograde, hétérodoxe, misogyne, etc.) dont la pensée réformiste tatar - puis le régime soviétique - a repris ensuite tous les pesants clichés. On sait comment l'aiguillon de ces missions et de leurs activités orientalistes a puissamment contribué au développement de l'islam jadîd, sur de nombreux points.

Une question de fond ne pouvait sans doute être traitée par ce livre, qui apporte pourtant des éléments intéressants dans un débat fondamental, tout simplement celui qui porte sur ce qu'est l'islam ou sur ce qu'il est censé être, ou encore ce qu'il est censé devoir être. Dans l'opinion commune, comme parfois dans l'une ou l'autre contribution de ce recueil, flotte l'idée discutable, venue des sources missionnaires mais aussi de l'hérésiographie musulmane (que Mojan Membrado est la seule à évoquer), qu'il existerait effectivement quelque part une orthodoxie musulmane sunnite facilement identifiable, à l'aune de laquelle il serait facile de mesurer le degré d'aberrations théologiques des uns ou des autres. Cela pose le problème de l'orthodoxie en islam: existe-t-elle? Où et quand? Qui l'a forgée ? Des débats virulents comme ceux qui ont toujours existé entre différentes branches de l'islam, mais aussi au sein de différentes branches (par exemple sur l'appréciation de l'hagiologie d'Ibn 'Arabî et sur son monisme) permettent-ils de trancher? Qu'est-ce, finalement, qu'être musulman ? Comment les musulmans sunnites ou chiites ont-ils perçu les différentes hétérodoxies, dans différentes régions, à différents moments, selon quelles références? Problèmes délicats, d'autant que les communautés présentées n'ont pas le même rapport à l'islam ou la même vision de leur propre « orthodoxie » ou conformité à une norme islamique : les Kabyles se perçoivent comme sunnites et malékites - ce qu'ils sont effectivement alors que certaines communautés Ahl-e haqq se déclarent effectivement non- 
musulmanes, tandis que d'autres se présentent comme des confréries mystiques musulmanes.

Il est en fait souvent question d'ésotérisme, dans ce volume, à tonalité soufie et parfois d'inspiration chiite: la domination ou non de l'ésotérisme face à l'exotérisme, le rapport à la Loi et son interprétation, sont des sujets aussi déterminants pour l'islam que pour le judaïsme, ce que les missionnaires n'ont probablement pas compris. L'approche en termes purement historicistes (mais en fait sans histoire) de nombreux missionnaires, dans une fascination des origines et dans une quête de causalités réductrices, conduisait nécessairement à une impasse : on se condamnait à ne pas voir que les Ahl-e Haqq étaient le fruit des invasions mongoles; que les idées d'Akbar, si liées qu'elles fussent à la société indienne, devaient beaucoup aux adaptations indiennes de l'ésotérisme soufi et se heurtaient à des courants issus d'une autre adaptation indienne de l'ésotérisme soufi ou prétendant lutter contre des influences chiites ; que l'islam des Bamoun était une variété tijânî particulière et non une mixture pagano-islamique, etc. L'orthodoxie islamique, au fond, est un mythe que l'on cherche ou dans le passé (Médine), ou dans un lieu (le Hijâz, surtout quand on en est très éloigné, comme les Javanais ou les Indiens), ou dans des textes qui redéfiniraient la norme (Ghazâlî par exemple). À partir de là, tout islam est islam des marges, et l'islam n'est alors qu'un long et indéfini processus d'islamisation jamais achevé.

Le regard normatif, lui-même changeant et varié selon les moments, les congrégations et même les personnes, des missions chrétiennes sur l'islam et ses différents courants parcourt tout le livre. Les missions auront contribué à donner, en quelque sorte, directement ou indirectement, des modèles d'islamisation et aussi d'islam, elles auront proposé de nouvelles références, variées, en matière de normativité islamique et furent l'un des aliments, parmi d'autres, du réformisme musulman. Elles contribuèrent puissamment à son succès et à son dynamisme, grâce à la construction d'une véritable science missionnaire où puisèrent les réformistes, ou dans des rencontres réelles qui laissèrent entrevoir un espace commun sécularisé (p.15) - que les réformistes réinterprétèrent le plus souvent, notamment au Moyen-Orient, au profit d'un primat de l'islam. 